\title{
Asthma-COPD overlap is not a homogeneous disorder: further supporting data
}

\author{
Luis Pérez-de-Llano ${ }^{1,4^{*}+}$, Borja G. Cosio $2,3+$ on behalf of the CHACOS study group
}

\begin{abstract}
Asthma-COPD ovelap (ACO) is an umbrella term that encompasses patients with COPD and eosinophilic inflammation (e-COPD) and smoking asthmatics with non-fully reversible airflow obstruction (SA). We compared the clinical characteristics and the inflammatory profile of e-COPD and SA. Patients classified as e-COPD were older and more often male and showed significantly impaired pulmonary function (likely explained by a heavier smoking habit). On the contrary, SA had more atopic features, more reversibility of airflow obstruction and higher lgE levels. The concentrations of IL-5, IL-13, IL-8, IL-6, TNF-a, IL17 in serum were similar between the 2 groups. However, Th2-related biomarkers (periostin, FeNO and blood eosinophils) shower higher median values in e-COPD patients. Our findings reinforce the notion that ACO is a heterogeneous disorder and, as a consequence, it might be unacceptable to offer the same treatment for two related but different conditions.
\end{abstract}

Keywords: Eosinophils, Periostin, COPD, Asthma, Asthma-COPD overlap, ACO

\section{Dear editor}

We have read with great interest the letter by Kolsum et al. [1] and we fully agree that eosinophilic COPD (eCOPD) patients have distinct characteristics compared to smoking asthmatics (SA) who develop non-fully reversible airflow obstruction. Both entities are commonly encompassed under an umbrella term [2], the so-called Asthma-COPD overlap (ACO), but, in the age of personalized medicine [3], it might be unacceptable to offer the same treatment for two related but different conditions. Studies that focus on identifying ACO's phenotypes are scarce, but Lange et al. found that individuals with $\mathrm{ACO}$ and asthma onset before the age of 40 years have better prognosis than those whose asthma starts after this age [4]. On the other hand, given that asthma and COPD are themselves heterogeneous diseases, one could argue whether it is necessary to define their overlap as a new entity. All these problems could be sorted out by identifying endotypes of obstructive lung disease

\footnotetext{
* Correspondence: eremos26@hotmail.com

${ }^{\dagger}$ Equal contributors

'Department of Respiratory Medicine, Hospital Lucus Augusti, Lugo, Spain

${ }^{4}$ Pneumology Service, Hospital Universitario Lucus Augusti, Calle Dr Ulises

Romero $n^{\circ}$ 1. 27003, Lugo, Spain

Full list of author information is available at the end of the article
}

(OLD) that would allow a personalized approach to therapy. In this regard, we have recently published a study that postulated the extinction of $\mathrm{ACO}$ and the use of a Th2 inflammation biomarker to differentiate a pooled population of patients with OLD [5]. With this letter, we would like to provide additional information to support the differentiation between e-COPD and SA.

We have performed a cross-sectional, observational, multicenter study carried out in 23 out-patient clinics from tertiary hospitals in Spain. The details of the design are described elsewhere [5]. Two hundred and ninetytwo patients with OLD were included in the study: 94 non-smoking asthmatics, 89 non-eosinophilic COPD, 44 $\mathrm{SA}$ and 65 e-COPD. All investigators were asked to prospectively recruit 12 consecutive eligible patients with OLD from their clinics.

Patients were labelled as SA if they had been previously diagnosed with asthma according to GINA guidelines [6] and, after having smoked $>20$ pack-years, they subsequently developed non-fully reversible airflow obstruction (FEV1/FVC $<70 \%$ post-bronchodilator). The diagnosis of e-COPD was made in patients who were previously diagnosed with COPD according to GOLD recommendations [7], in the absence of a clinical 
suspicion for asthma and in the presence of a blood eosinophil count $>200$ eosinophils $/ \mu \mathrm{l}$.

There were important differences between patients with SA and e-COPD (Table 1). Patients classified as e-COPD were older and significantly more often male. They showed significantly lower post-bronchodilator FEV1 than SA patients $(54.49 \pm 15.2$ vs $65.57 \pm 17.5 \%$; $p=0.005)$ and lower DLCO values $(64.0 \pm 19.9$ vs $70.0 \pm 17.6 \% ; p<0.001)$, likely due to a heavier smoking habit $(51.8 \pm 28$ vs $35,1 \pm 13.2$ pack-year; $\mathrm{p}<0.001)$. On

Table 1 Demographics, clinical and functional characteristics of patients

\begin{tabular}{|c|c|c|c|}
\hline Variables $^{a}$ & $\begin{array}{l}\text { Smoking } \\
\text { asthmatics }\end{array}$ & e-COPD & $P$ Value \\
\hline Number of subjects & 44 & 65 & \\
\hline Age, yrs & $59.8(10.5)$ & $65.6(10.1)$ & 0.007 \\
\hline Gender (\% female) & 59.1 & 18.5 & $<0.001$ \\
\hline BMI $\left(\mathrm{Kg} / \mathrm{m}^{2}\right)$ & $28.2(5.2)$ & $28.8(6.5)$ & 0.91 \\
\hline Pack/yrs ${ }^{b}$ & $35.1(13.2)$ & $51.8(28)$ & $<0.001$ \\
\hline Age of onset (yrs) & $48.5(19.1)$ & $53.6(12.3)$ & $<0.001$ \\
\hline $\mathrm{SPT}^{\mathrm{C}}(\%)$ & 45.5 & 23.1 & 0.003 \\
\hline Rhinitis (\%) & 51.2 & 17.5 & 0.001 \\
\hline $\begin{array}{l}\text { Patients with nocturnal } \\
\text { symptoms (\%) }\end{array}$ & 39.5 & 9.5 & 0.001 \\
\hline Comorbidities: & 38.6 & 47.7 & 0.36 \\
\hline $\begin{array}{l}\text {-Arterial hypertension (\%) } \\
\text {-Diabetes (\%) }\end{array}$ & 9.1 & 23.1 & 0.26 \\
\hline -Ischemic heart disease (\%) & 4.5 & 4.6 & 0.11 \\
\hline -Heart failure (\%) & 1.0 & 7.7 & 0.26 \\
\hline -Anemia (\%) & 0 & 1.5 & 0.58 \\
\hline -Osteoporosis (\%) & 18.6 & 3.2 & 0.002 \\
\hline -Psychiatric disorders (8\%) & 18.2 & 4.6 & 0.15 \\
\hline $\begin{array}{l}\text {-Gastro-esophageal } \\
\text { Reflux (\%) }\end{array}$ & 35.7 & 12.7 & $<0.001$ \\
\hline Prebd FEV1 ${ }^{\mathrm{d}}(\%)$ & $59.0(18.3)$ & $50.3(14.3)$ & $<0.001$ \\
\hline Posbd FEV1 ${ }^{\mathrm{e}}(\%)$ & $65.5(17.5)$ & $54.5(15.2)$ & 0.001 \\
\hline $\mathrm{PBT}^{\mathrm{f}}(\%)$ & 48.8 & 30.2 & 0.001 \\
\hline $\operatorname{DLCO}^{9}(\%)$ & $70.0(17.6)$ & $64.0(19.9)$ & $<0.001$ \\
\hline Exacerbations ${ }^{h}$ & $1.00(1.3)$ & $0.95(1.3)$ & 0.62 \\
\hline $\lg E(I U / m l)$ & $112(4,1340)$ & $76(5,2500)$ & 0.005 \\
\hline CAT $^{\mathrm{i}}$ & $13.2(8.0)$ & $13.4(7.7)$ & 0.83 \\
\hline$A C T^{j}$ & $19.5(4.9)$ & $19.2(4.8)$ & 0.54 \\
\hline \multicolumn{4}{|c|}{ 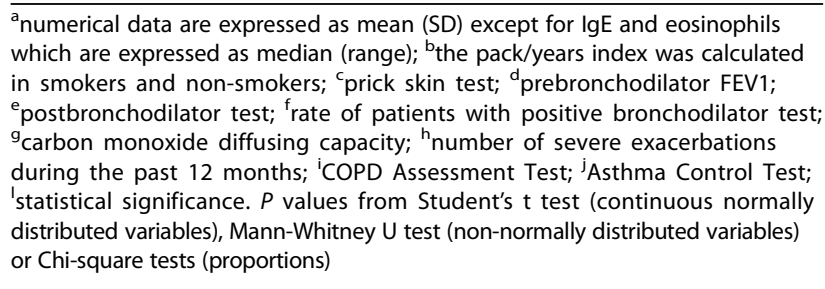 } \\
\hline
\end{tabular}

the contrary, SA had more atopic features, more reversibility of airflow obstruction and higher IgE levels. There were no significant differences between the 2 groups with respect to symptoms and exacerbations. Detailed demographic, clinical and functional information is displayed in Table 1. Part of these data have been already published elsewhere [5].

These results are well in accordance with those found by Kolsum et al., and the most remarkable difference is that we were unable to observe any difference between groups in the exacerbation rate in the 12 months prior to study entry, while they found AS to be more prone to suffer exacerbations. To explain this discordance, we must take into account that our patients were fairly well-controlled as assessed by the CAT and ACT questionnaires and the low exacerbation rate.

In order to obtain more detailed information about the underlying inflammatory pattern of OLD patients, we have measured Th-2 characteristic biomarkers such as fractional exhaled nitric oxide (FeNO), blood eosinophils, periostin (DuoSet ELISA, R\&D Systems, Minneapolis, MN, USA) and the concentrations of IL-5 and IL-13 in serum. Additionally, serum levels of cytokines representative of systemic inflammation (TNF $\alpha$ and IL-6) and those associated with a neutrophilic inflammatory response (IL-17 and IL-8) were determined (Merck Millipore ${ }^{\circ}$ ). The levels of these cytokines were similar between SA and eCOPD groups. However, Th2-related biomarkers (periostin, FeNO and blood eosinophils) showed higher median values in e-COPD patients (Table 2). Approximately $49 \%$ of e-COPD patients and 30\% of SA showed a "Th-2 high" inflammatory pattern (defined as eosinophil count $>300$ eosinophils $/ \mu \mathrm{L}$ in blood or $\geq 3 \%$ in sputum) ( $p=0.02)$. Again, these results are consistent with the ones found by Kolsum et al. Interestingly, they observed that only $35.7 \%$ of SA patients had blood eosinophil counts $\geq 300$ cells/ $\mu$ l (a similar

Table 2 Differences in the inflammatory profile between the 2 groups

\begin{tabular}{llll}
\hline & $\begin{array}{l}\text { Smoking asthmatics } \\
n=44\end{array}$ & e-COPD $n=65$ & $p$-value \\
\hline IL-13 & $1.98(0.23-3.92)$ & $2.18(0.23-4.20)$ & 0.352 \\
IL-17 & $7.26(2.37-16.35)$ & $6.67(3.12-10.81)$ & 0.142 \\
IL-5 & $1.63(0.40-2.77)$ & $1.75(0.42-2.85)$ & 0.371 \\
IL-6 & $1.37(0.42-3.28)$ & $1.43(0.67-4.12)$ & 0.426 \\
IL-8 & $9.54(6.40-14.20)$ & $9.68(6.25-12.98)$ & 0.652 \\
TNF & $3.22(2.19-4.32)$ & $4.00(3.19-5.07)$ & 0.740 \\
Periostin & $30.8(23.3-38)$ & $39.7(30-49.2)$ & 0.005 \\
FENO & $19.6(10-19.9)$ & $24.5(10-24.6)$ & $<0.001$ \\
Blood Eos (cels/ $\mu$ l) & $200(0,1000)$ & $300(210,940)$ & $<0.001$ \\
\hline $\begin{array}{l}\text { Numerical data are expressed as median and (interquartile range) except for } \\
\text { eosinophils which are expressed as median (range). } P \text { values from Mann-Whitney } \\
\text { U test } \\
p \text {-values }\end{array}$ & &
\end{tabular}


percentage to our $30 \%$ ) and only $46 \%$ of them showed sputum eosinophil counts $\geq 3 \%$. What seems clear is that SA forms a more heterogeneous group from the inflammatory point of view- than e-COPD, probably encompassing Th-2 high, neutrophilic and mixed endotypes. This could be explained by the fact that SA definition relies on clinical characteristics (asthma diagnosis and smoking history) whereas e-COPD definition includes a biomarker of Th-2 inflammation. In fact, periostin and FENO were higher in the e-COPD group compared to SA and it would also have been expected to find higher values of Th2-related cytokines (IL-5, IL-13), but we did not perceive statistically significant differences, although a tendency could be observed (Table 2). These discordant results could be justified by an insufficient sample size or by the lack of association between lung and serum biomarkers reported by several authors, both in COPD $[8,9]$ and asthma patients [10]. It has been shown that the correlation between blood and sputum eosinophilia becomes weaker in those patients with severe asthma and it has been hypothesized that type 2 innate lymphocytes, a steroid-resistant cell, able to produce eosinophilopoietic factors, could explain the persistence of sputum eosinophils in patients with normal blood counts [10]. If such a discrepancy exists, it would not be surprising to find it in other inflammatory mediators such as Th2-related cytokines. A different approach would be to classify ACO patients according to the inflammatory pattern (specific endotypes) in order to evaluate, in a second step, whether it might result in clinical differences. However, we have found poor correlation between the inflammatory markers, which hinders the possibility of defining distinct inflammatory clusters. For example, correlations between IL-5 and IL-13, between IL-5 and blood Eos and between IL-5 and FENO were 0.4, 0.06 and 0.1 respectively in SA patients $(0.3,0.03$ and $0-1$ in e-COPD patients).

It has been largely debated whether asthma and COPD are distinct entities generated by different mechanisms [11] or, on the contrary, they are in fact expressions of one basic disease in which the combined endogenous (host) and exogenous (environmental) factors shape the patient's clinical profile [12]. Irrespective of that, a current perspective of classification and therapeutic management of OLD patients should take into account the growing knowledge on molecular pathways that has allowed the development of novel therapeutic strategies that target specific components of the underlying inflammatory process. Previous attempts to define ACO $[2,6,7,13]$ do not consider the biological heterogeneity that we, and Kolsum et al., have found, which might potentially lead to inadequate therapeutic approaches. Any useful definition of ACO should offer guidance to make therapeutic decisions, particularly to effectively select who of them can benefit from inhaled corticosteroids treatment (or even anti-Th2 biological drugs in the near future).

In conclusion, our findings reinforce the notion that $\mathrm{ACO}$ is a heterogeneous disorder. In fact, OLD is heterogeneous and classical diagnostic categories are unable to fully explain the great complexity of the underlying inflammatory process that ultimately determines the response to treatment. Therefore, we must advance step by step towards a more personalized medicine.

\section{Acknowledgements \\ CHACOS collaboration group: Amanda Iglesias ${ }^{1}$, Natividad de las Cuevas², Juan Jose Soler-Cataluña ${ }^{3,1}$, Jose Luis Izquierdo ${ }^{4}$, Jose Luis López-Campos ${ }^{5,1}$, Carmen Calero ${ }^{6}$, Vicente Plaza ${ }^{6,1}$, Marc Miravitlles ${ }^{7,}$, Alfons Torrego ${ }^{6}$, Eva Martínez Moragón ${ }^{8}$, Joan B Soriano ${ }^{9}$, Antolin Lopez Viña ${ }^{10}$ and Irina Bobolea ${ }^{11}$. \\ All of them gave the authors permission to acknowledge. \\ 1. CIBER de Enfermedades Respiratorias (CIBERES). Instituto de Salud Carlos III, Madrid \\ 2. Department of Allergy, hospital 12 de Octubre, Madrid, Spain \\ 3. Department of Respiratory Medicine, hospital Arnau de Vilanova, Valencia, Spain \\ 4. Department of Respiratory Medicine, hospital Universitario de Guadalajara, \\ Guadalajara, Spain \\ 5. Department of Respiratory Medicine, Hospital Virgen del Rocío, Sevilla, Spain. 6. Department of Respiratory Medicine, Hospital de la Santa Creu y Sant Pau, Barcelona; Institut d'Investigació Biomédica Sant Pau (IIB Sant Pau); Universitat Autònoma de Barcelona, Department of Medicine, Barcelona, Spain. \\ 7. Department of Respiratory Medicine, Hospital Universitari Vall d'Hebron, Barcelona, Spain. \\ 8. Department of Respiratory Medicine, Hospital Dr. Peset, Valencia, Spain. \\ 9. Instituto de Investigación Hospital de la Princesa, Universidad Autónoma de Madrid, Madrid, Spain. \\ 10. Department of Respiratory Medicine, Hospital Puerta de Hierro, Madrid, Spain. 11. Servei de Pneumologia i Alergia. Hospital Clinic. Barcelona.}

\section{Funding}

The project was endorsed by the COPD and Asthma Research Board (PII de EPOC $y$ asma) of the Spanish Society of Pneumology and Thoracic Surgery (SEPAR). The project was partially funded by the Fondo de Investigaciones Sanitarias, Instituto de Salud Carlos III, Ministerio de Economia y

Competitividad (FIS 15/01263) and by an unrestricted grant from Chiesi España S.A.U.

\section{Availability of data and materials}

The datasets used and/or analysed during the current study are available from the corresponding author on reasonable request.

\section{Authors' contibutions}

LPLL and BC made substantial contributions to conception and design of the study, and analysis and interpretation of data. They have both been involved in drafting the manuscript and revising it critically for important intellectual content. Both authors have read and approved the final manuscript.

\section{Ethics approval and consent to participate}

The data presented here were extracted from a cross-sectional, observational, multicenter study carried out in 23 out-patient clinics from tertiary hospitals in Spain run by expert respiratory physicians. All participants gave a signed informed consent. The study was approved by the Research Ethics Committee of the Balearic Islands (Cod: IB2499/15). Additionally, an independent Ethics committee or institutional review board for each study centre approved the final protocol.

Consent for publication

A consent form will be be made available to the Editor if requested.

Competing interests

The authors declare that they have no competing interests. 


\section{Publisher's Note}

Springer Nature remains neutral with regard to jurisdictional claims in published maps and institutional affiliations.

\section{Author details}

'Department of Respiratory Medicine, Hospital Lucus Augusti, Lugo, Spain.

${ }^{2}$ Department of Respiratory Medicine, Hospital Universitario Son Espases-IdISBa, Palma de Mallorca, Spain. ${ }^{3}$ CIBER de Enfermedades Respiratorias (CIBERES), Instituto de Salud Carlos III, Madrid, Spain. ${ }^{4}$ Pneumology Service, Hospital Universitario Lucus Augusti, Calle Dr Ulises Romero $n^{\circ}$ 1. 27003, Lugo, Spain.

Received: 23 June 2017 Accepted: 17 October 2017

Published online: 02 November 2017

\section{References}

1. Kolsum U, Ravi A, Hitchen P, Maddi S, Southworth T, Singh D. Clinical characteristics of eosinophilic COPD versus COPD patients with a history of asthma. Respir Res. 2017 Apr 26;18(1):73.

2. Miravitlles M, Alvarez-Gutierrez FJ, Calle M, Casanova C, Cosio BG, LópezViña A, Pérez de Llano L, Quirce S, Roman-Rodríguez M, Soler-Cataluña JJ, Plaza V. Algorithm for identification of asthma-COPD overlap: consensus between the Spanish COPD and asthma guidelines. Eur Respir J. 2017 May 1:49(5):1700068

3. Agusti A. The path to personalised medicine in COPD. Thorax. 2014 September; 69(9):857-64.

4. Lange $P$, Çolak $Y$, Ingebrigsten TS, Vetsbo J, Marott JL. Long-term prognosis of asthma, chronic obstructive pulmonary disease, and asthma-chronic obstructive pulmonary disease overlap in the Copenhagen City heart study: a prospective population-based analysis. Lancet Respir Med. 2016;4(6):454-62

5. Cosío BG, Pérez de Llano L, López Viña A, et al. Th-2 signature in chronic airway diseases: towards the extinction of asthma-COPD overlap syndrome? Eur Respir J. 2017:49:1602397.

6. Global Strategy for Asthma Management and Prevention, Global Initiative for Asthma (GINA) 2015. Available from: ginasthma.org/2017-gina-reportglobal-strategy-for-asthma-management-and-prevention/. 2016.

7. Vestbo J, Hurd SS, Agusti AG, Jones PW, Vogelmeier C, Anzueto A, et al. Global strategy for the diagnosis, management, and prevention of chronic obstructive pulmonary disease: GOLD executive summary. Am J Respir Crit Care Med. 2013 February 15;187(4):347-65

8. Ropcke S, Holz O, Lauer G, Muller M, Rittinghausen S, Ernst P, et al. Repeatability of and relationship between potential COPD biomarkers in bronchoalveolar lavage, bronchial biopsies, serum, and induced sputum. PLoS One. 2012;7(10):e46207.

9. Nunez B, Sauleda J, Garcia-Aymerich J, Noguera A, Monso E, Gomez F, et al. Lack of correlation between pulmonary and systemic inflammation markers in patients with chronic obstructive pulmonary disease: a simultaneous, two-compartmental analysis. Arch Bronconeumol. 2016 July:52(7):361-7.

10. Mukherjee $M$, Nair P. Blood or sputum eosinophils to guide asthma therapy? Lancet Respir Med. 2015 November;3(11):824-5.

11. Vermeire PA, Pride NBA. "Splitting" look at chronic nonspecific lung disease (CNSLD): common features but diverse pathogenesis. Eur Respir J. 1991;4: 490-6.

12. Orie NGM, Sluiter HJ. Bronchitis: an international symposium. Assen, Netherlands: Royal van Gorcum; 1962.

13. Sin DD, Miravitlles M, Mannino DM, et al. What is asthma-COPD overlap syndrome? Towards a consensus definition from a roundtable discussion. Eur Respir J. 2016;48:664-73.

\section{Submit your next manuscript to BioMed Central and we will help you at every step:}

- We accept pre-submission inquiries

- Our selector tool helps you to find the most relevant journal

- We provide round the clock customer support

- Convenient online submission

- Thorough peer review

- Inclusion in PubMed and all major indexing services

- Maximum visibility for your research

Submit your manuscript at www.biomedcentral.com/submit 